\title{
Enteric Coated Oral Delivery of Hydroxyapatite Nanoparticle for Modified Release Vitamin $D_{3}$ Formulation
}

\author{
Ranga K. Dissanayake $\mathbb{D}^{1}$ K. D. C. Perera, ${ }^{2}$ W. P. T. D. Perera $\mathbb{D}^{2}$ W. P. S. L. Wijesinghe, ${ }^{2}$ \\ and Janitha M. Unagolla ${ }^{3}$ \\ ${ }^{1}$ Department of Pharmacy and Pharmaceutical Sciences, Faculty of Allied Health Sciences, University of Sri Jayewardenepura, \\ Gangodawila, Nugegoda 10250, Sri Lanka \\ ${ }^{2}$ Sri Lanka Institute of Nanotechnology, Nanotechnology and Science Park, Mahenwatte, Pitipana, Homagama 10206, Sri Lanka \\ ${ }^{3}$ Department of Bioengineering, College of Engineering, University of Toledo, Toledo, $\mathrm{OH}$ 43607, USA
}

Correspondence should be addressed to Ranga K. Dissanayake; rangad@sjp.ac.lk

Received 25 March 2021; Revised 1 November 2021; Accepted 9 November 2021; Published 15 December 2021

Academic Editor: Hassan Azzazy

Copyright (C) 2021 Ranga K. Dissanayake et al. This is an open access article distributed under the Creative Commons Attribution License, which permits unrestricted use, distribution, and reproduction in any medium, provided the original work is properly cited.

\begin{abstract}
Vitamin $\mathrm{D}_{3}$ (VD) and calcium phosphate play a vital role in bone homeostasis. Factors such as obesity or gastrointestinal problems can render the use of pure VD and calcium phosphate supplements ineffective. This study investigated the possibility of using VD-loaded hydroxyapatite nanoparticles for the codelivery of $\mathrm{VD}$ and $\mathrm{Ca}_{3}\left(\mathrm{PO}_{4}\right)_{2}$. Due to the high affinity of $\mathrm{Ca}_{3}\left(\mathrm{PO}_{4}\right)_{2}$ for bone tissue, $\mathrm{HA}$ is an ideal delivery system to deliver VD to target tissue. Herein, HA nanoparticles were synthesized and loaded with VD using a vacuum evaporation method. The synthesized HA-VD nanoparticles were morphologically and chemically characterized by SEM, FTIR, and TGA. The system exhibited a two-stage release pattern, which includes a first-day burst release (35\%) and sustained release for a further ten days. The cytocompatibility and cell penetrative ability of the nanoparticle system were assessed in vitro using preosteoblast cells: the system is nontoxic and well-tolerated. Finally, the VD-loaded HA nanoparticles were coated with a gastroresistant polymer, hypromellose phtalate-55 (HP-55) in order to protect the $\mathrm{pH}$-sensitive HA from degradation at lower pHs. A coaxial electrospray technique was employed to achieve this. In all, the tested HA-VD system is a viable alternative for codelivery of $\mathrm{VD}, \mathrm{Ca}^{2+}$, and $\mathrm{PO}_{4}{ }^{3-}$ to their target tissues.
\end{abstract}

\section{Introduction}

Vitamin D is a lipophilic group of vitamins with a steroidal origin. Though there are five types of vitamin $\mathrm{D}$ (vitamins $\mathrm{D}_{1}-\mathrm{D}_{5}$ ), the most important are cholecalciferol (vitamin $\mathrm{D}_{3} /$ VD) and ergocalciferol (vitamin $\mathrm{D}_{2}$ ). Vitamin $\mathrm{D}_{3}$-which exists in dietary supplement formulations-is the major type over the plant-derived $\mathrm{VD}_{2}[1,2]$. For the majority of the population, however, the principal source of vitamin $\mathrm{D}$ is synthesis following exposure of the skin to UVB radiation, converting 7-dehydrocholesterol to the provitamin cholecalciferol $[1,3]$.

Vitamin D plays a vital role in in vivo homeostasis and metabolism, as well as heavily affecting bone growth. Obese individuals have a higher risk towards developing inadequate serum VD concentrations due to its accumulation in the adipose tissue, which serves as a reservoir of VD $[4,5]$. Thus, a delivery system of pure VD is not a suitable method for the correction of VD deficiency, since most of the administrated VD tends to accumulate naturally inside adipocytes. In addition, regular administration of such formulations may cause VD-related toxicity [6, 7]. The solution is a controlledrelease VD delivery system with a high affinity to target sites. There are several existing targeted delivery systems for VD, such as micelles, dendrimers, liposomes, lipidic nanoparticles, and carbon and silicon nanotubes [7-9].

Calcitriol, the metabolite that is the active form of vitamin D, expresses its biological activities by binding to the vitamin $\mathrm{D}$ receptor (VDR), which is located chiefly in the nuclei of target cells. VDRs are found in many tissues of the body including the skin, bone, muscle (both skeletal 
and cardiac), and endocrine, as well as throughout the immune system $[10,11]$. Calcitriol-bound VDRs act as transcription factors that initiate gene expression to produce transport proteins such as TRPV6 and calbindin, which are involved in calcium absorption in the intestine. Due to their lipophilic nature, VD absorption is blocked when there is insufficient lipids in the intestine. To improve its bioavailability, it is important to enhance its water solubility [12-14].

Most cases of VD deviancies are associated with deficiencies of calcium and phosphorus due to a lack of mechanisms to absorb these ions through the gut epithelium. Codelivery of VD with calcium and phosphate can potentially resolve this problem $[15,16]$. Recently, hydroxyapatite $\left(\mathrm{HA} ; \mathrm{Ca}_{10}\left(\mathrm{PO}_{4}\right)_{6}(\mathrm{OH})_{2}\right)$ has enjoyed heightened attention in the biomedical field due to its exceptional features in biocompatibility, bioactivity, osteoconductivity, and osteoinductivity $[8,17,18]$. The use of VD-loaded HA nanoparticles resolves most of the problems associated with both vitamin $\mathrm{D}-, \mathrm{Ca}^{2+}$, and $\mathrm{PO}_{4}{ }^{3-}$ deficiencies.

In this study, a laboratory-synthesized composite of hydroxyapatite nanoparticles loaded with vitamin $\mathrm{D}_{3}$ has been evaluated as a possible delivery system of vitamin $\mathrm{D}$ with a sustained release profile. The synthesis and morphology of the HA nanoparticles are confirmed with electron micrographs. The loading of VD is confirmed by thermal analysis and FTIR data together with assessment of drug release kinetics. Finally, toxicological and cell penetration profiles of HA and HA-VD systems were evaluated in vitro using a murine preosteoblast cell line.

\section{Materials and Methods}

2.1. Materials. Calcium sucrate, ammonium dihydrogen orthophosphate, cholecalciferol, phosphate-buffered saline, ethanol, and WST-1 (Roche diagnostic) were all purchased from Sigma-Aldrich Chemicals (St. Louis, MO, USA). Alpha minimum essential media ( $\alpha$-MEM), fetal bovine serum (FBS), phosphate buffered saline (PBS), Dulbecco's phosphate buffered saline (DPBS), and penicillin/streptomycin were purchased from Gibco (Thermo Fisher Scientific, USA). Live/dead cell viability/cytotoxicity kit was purchased from Invitrogen (USA).

\subsection{Methods}

\subsubsection{Particle Synthesis}

(1) Preparation of HA Nanoparticles. First, a $0.50 \mathrm{M}$ calcium sucrate solution was prepared according to our previously published method. The required amount of $\mathrm{CaO}$ was added to the sucrose solution followed by stirring for $12 \mathrm{~h}$. Calcium sucrate was then reacted with ammonium dihydrogen orthophosphate to synthesize HA. The Ca:P mole ratio was kept at $1.67: 1$. The mixture was stirred for $24 \mathrm{~h}$, and our previously published method was modified by hydrothermally treating the resulting $\mathrm{HA}$ precipitate at $150^{\circ} \mathrm{C}$ for $12 \mathrm{~h}$. Finally, precipitates were centrifuged at $5000 \mathrm{rpm}$ for $15 \mathrm{~min}$ and washed with $50.0 \mathrm{~mL}$ of distilled water thrice in order to remove impurities. The products were dried in a vacuum oven at $60^{\circ} \mathrm{C}, 600 \mathrm{~mm} \mathrm{Hg}$ for $12 \mathrm{~h}[19,20]$.
(2) Preparation of VD-Loaded HA Nanoparticles. VD was composited with prepared HA nanoparticles using a standard vacuum evacuation process. HA (2 g) and VD (250 $\mathrm{mg}$, maintaining an $8: 1 \mathrm{w} / \mathrm{w}$ ratio) were dispersed in $10 \mathrm{~mL}$ of ethanol for $1 \mathrm{~h}$ at $600 \mathrm{rpm}$ followed by ultrasonication for 15 min. The flask containing the resultant HA-VD suspension was evacuated using a vacuum pump for 10 min until a slight fizzing of the suspension was observed, indicating the removal of entrapped air. After the fizzing stopped, the suspension was kept uninterrupted for $10 \mathrm{~min}$ to reach equilibrium, and the entire vacuum evacuation cycle was repeated thrice to promote VD's inclusion with HA. Following this, the suspension was centrifuged and rinsed twice using ethanol to remove excess VD. The UV absorbance of the supernatant of the rinsed HA-VD was measured at $275-280 \mathrm{~nm}$, and the encapsulation percentage was calculated based on a standard calibration curve of VD [21].

(3) Preparation of HP-55 Coated HA-VD Nanoparticles. The electrospray system consisted of a high voltage power supply, a coaxial needle (Linari Nanotech; inner and outer needle diameters of $0.5 \mathrm{~mm}$ and $0.6 \mathrm{~mm}$, respectively), two syringe pumps, and a collector. The distance between positive electrode (needle) and the negative electrode (collector) was $20 \mathrm{~cm}$, and the applied voltage was $12.0 \mathrm{kV}(0.02 \mu \mathrm{A})$. The core solution of the coaxial system contained aqueous suspension of $10 \%(w / v)$ HA-VD NPs while the shell solution contained $5 \%(w / v)$ methanolic solution of HP-55 polymer (prepared at $\mathrm{pH}>10$ ). The two solutions were sprayed to $0.2 \mathrm{M} \mathrm{HCl}$ solution with flow rates of $0.05 \mathrm{~mL} / \mathrm{h}$ (core) and $0.11 \mathrm{~mL} / \mathrm{h}$ (shell). The synthesized particles were collected by centrifuging and washing at $1500 \mathrm{rpm}[22,23]$.

\subsubsection{Characterization of Nanoparticles}

(1) Morphological and Thermal Analysis. The morphologies of nanoparticles synthesized using the methods set out above were evaluated using field-emission scanning electron microscopy (SEM) (Hitachi SU6600 setup) and transmission electron microscopy (TEM) (Jeol 2100). All SEM samples were subjected to gold sputtering prior to analysis. Energy dispersive X-ray (EDX) spectroscopy studies were carried out to confirm the VD impregnation onto the walls of HANPs with a scanning rate of 192000 CPS for $4.5 \mathrm{~min}$.

The thermal stabilities of the synthesized HA-NP and HA-VD were determined by thermogravimetric analysis (TGA) (STD Q600 setup) over a temperature range of 25 to $1000^{\circ} \mathrm{C}$ at a ramp of $20^{\circ} \mathrm{C} / \mathrm{min}$ in a nitrogen medium.

(2) Chemical Characterization. Fourier-transform infrared (FT-IR) spectroscopic analysis was performed in order to confirm VD loading onto HA nanoparticles and HP-55 coating. All spectra were obtained over the $4000-500 \mathrm{~cm}^{-1}$ region with 32 scans per measurement at a resolution of $4 \mathrm{~cm}^{-1}$ using a Bruker Vertex 80 Fourier-transform infrared spectrophotometer (Bruker, USA). The spectrophotometer was equipped with a L-alanine doped triglycine sulfate 
(DLaTGS) detector and MIRacle single-reflection horizontal attenuated total reflectance (ATR) accessory (PIKE Technologies, USA) working at room temperature.

2.2.3. Drug Release Studies. The release profile of VD from the enteric-coated HA-VD composite was assessed in phosphatebuffered saline (PBS) $(\mathrm{pH}=7.4)$ containing $0.5 \%(w / v)$ sodium azide (to prevent microbial contamination). $200 \mathrm{mg}$ of $\mathrm{HA}-\mathrm{VD}$ was added to $5 \mathrm{~mL}$ of $\mathrm{PBS}$ at $30^{\circ} \mathrm{C}$ under $200 \mathrm{rpm}$ stirring. At predetermined time points, $0.2 \mathrm{~mL}$ aliquots of the sample were removed and diluted with $0.8 \mathrm{~mL}$ of ethanol (1:5 dilution). The concentration of VD was determined by absorbance measurements at $275-280 \mathrm{~nm}$ (Shimadzu UV3600 UV - Vis-NIR spectrophotometer) with respect to a preprepared standard curve $[24,25]$. In order to eradicate the effect of HP-55 for UV-vis absorption, 5\% (w/v) HP-55 dissolved in $\mathrm{pH} 10.0$ buffer solution was used as the control. After the desired period, the mixture was sonicated for $2 \mathrm{~h}$ at room temperature to establish the loading capacity of VD. The cumulative drug release was assessed using equation (1) where $W_{i}$ is the weight of the VD in the solution and $W_{t}$ is the total VD of added nanomaterial.

$$
\text { Cumulative release rate }(\%)=\frac{W_{i}}{W_{t}} \times 100 \text {. }
$$

(1) Loading Capacity of VD. $200 \mathrm{mg}$ of enteric coated HA-VD material was mixed with $5 \mathrm{~mL}$ of $\mathrm{pH} 9$ borate buffer solution in order to remove the coating. After $15 \mathrm{~min}$ with continuous stirring, the resultant suspension was centrifuged at $3000 \mathrm{rpm}$ for $10 \mathrm{~min}$, and the pellet was dissolved in $5 \mathrm{~mL}$ of $0.1 \mathrm{M} \mathrm{HCl}$ solution with continuous stirring for $2 \mathrm{hrs}$. The released amount of VD was quantified using the previously described UV-vis spectrophotometric technique with respect to a preprepared standard curve (buffer as control). The loading capacity was calculated using equation (2).

Loading capacity $(\%)=\frac{\text { Weight of the drug in nanoparticle }}{\text { Weight of the nanoparticles }} \times 100$.

\subsubsection{Cytocompatibility and Cell Penetration Studies}

(1) Nanomaterial Preparation and Cell Seeding. The murine preosteoblast cell line OB6 was cultured in $\alpha$-MEM medium containing $15 \% \quad(v / v) \quad$ FBS and $1 \% \quad(v / v)$ penicillinstreptomycin. The culture was maintained in a humidified incubator at $37^{\circ} \mathrm{C}$ with $5 \% \mathrm{CO}_{2}$, and the culture medium was replaced every 3 days. After $80 \%$ confluency was reached, the cells were subcultured using $0.25 \%(v / v)$ trypsin-EDTA solution. A cell density of $1 \times 10^{4}$ was used to seed each well of a 24-well plate which contained HA and HA-VD, with a seed volume of $300 \mu \mathrm{L}$ per well, and incubated for $3 \mathrm{~h}$ to facilitate initial cell adhesion. Following the $3 \mathrm{~h}$ incubation, $700 \mu \mathrm{L}$ of the $\alpha$-MEM was added to the each.

(2) Live Cell Assay. Live cell assay was performed for cell seeded nanomaterials at day 3 , according to the manufacturer's protocol. Briefly, $5 \mu \mathrm{L}$ of $4 \mathrm{mM}$ calcine was added to
$10 \mathrm{~mL}$ of $1 \mathrm{x}$ DPBS to make the live assay solution. After removing the $\alpha$-MEM growth medium from each well, wells were washed with $1 \mathrm{x}$ PBS twice. Then, $300 \mu \mathrm{L}$ of prepared live assay solution was added to each well containing nanoparticles and incubated at room temperature for $15 \mathrm{~min}$. The cells were observed using cell imaging fluorescence microscopy for cell distribution and proliferation with nanoparticles.

(3) Cell Viability Assay. The water-soluble tetrazolium salt (WST-1) assay was used to measure cell viability after nanomaterial application. After standard $24 \mathrm{~h}$ incubation, all media in the wells were removed, and the wells washed with PBS. Fresh $\alpha$-MEM medium was added to each well with $10 \%(v / v)$ WST-1 reagent. The well plates were shaken for $2 \mathrm{~min}$ at $300 \mathrm{rpm}$ for homogeneous mixing of WST-1 with the $\alpha$-MEM medium. The plate was then incubated for $6 \mathrm{~h}$ at $37^{\circ} \mathrm{C}$ and $5 \% \mathrm{CO}_{2}$. Following this, $100 \mu \mathrm{L}$ from each well was transferred to a 96-well plate and absorbance readings taken using a UV spectrophotometer (SpectraMax190, Molecular Devices) at a wavelength of $440 \mathrm{~nm}$. All experiments were done in triplicate, and mean cell viabilities were calculated against the negative control (cells only). Blank wells that contained nanomaterials without cells were also assessed [26, 27].

(4) Cell Penetration of $H A-V D$. The cell penetration and accumulation ability of HA-VD were evaluated using the same preosteoblast cell line used for cytotoxicity studies. A cell density of $1 \times 10^{4}$ in $700 \mu \mathrm{L}$ of $\alpha$-MEM was seeded each well of a 24-well plate which contained $300 \mu \mathrm{L} \alpha$-MEM/HAVD suspension (10 mg of HA-VD). After $24 \mathrm{~h}$ incubation, unused medium was removed with serial washing without disturbing the cellular portion. The cells were then treated with $1 \mathrm{~mL}$ of $1 \mathrm{M} \mathrm{HCl}$ containing $0.01 \%(v / v)$ Triton X-100 and sonicated for $30 \mathrm{~min}$ at $70^{\circ} \mathrm{C}$ to destroy all cellular structures. The calcium ion concentration of supernatant was determined by ICP-MS with respect to a preprepared standard curve of $\mathrm{Ca}^{2+}$. The same procedure was repeated for cells without nanoparticles [28-30].

\section{Results and Discussion}

3.1. Morphological Properties. The SEM image of HA nanoparticles is provided in Figures 1(a) and 1(b) while TEM image is given in Figure 1(c). The synthesized HA particles were observed to be of a rod shape. HA nanoparticles were observed to possess an average diameter of approximately $20-60 \mathrm{~nm}$ and length of approximately $100-250 \mathrm{~nm}$. Thus, the successful synthesis of HA nanoparticles was confirmed by SEM and TEM imaging.

HP-55 coated electrospray HA-VD nanoparticles are shown in Figures 2(a) and 2(b). According to the surface morphology, the nanoparticles possess a smooth outer surface, and the HA-NPs are well covered with HP-55. The coating layer was detected by element mapping and EDX analysis which is provided in Figures 2(c) and 2(d). Therefore, the required protection from gastric acid can easily achieved by this coating. Electrospray as a technique has several advantages over conventional coating systems such as 


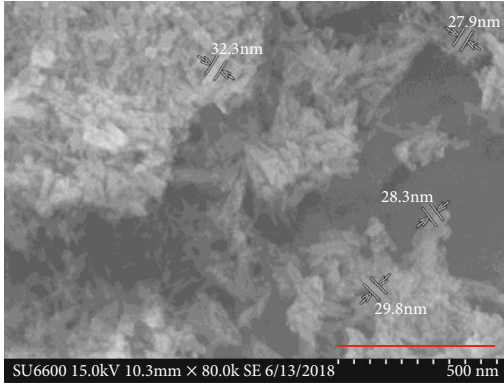

(a)

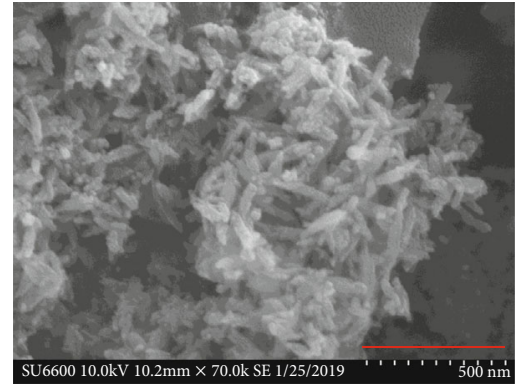

(b)

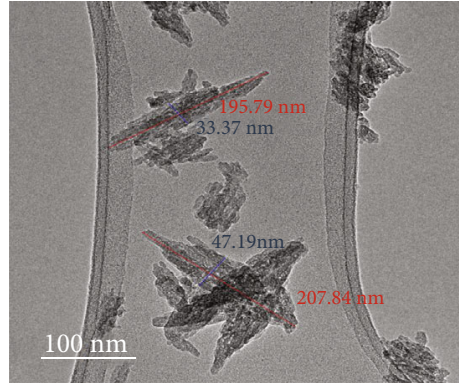

(c)

Figure 1: (a, b) SEM images of HA nanoparticles. (c) TEM image of HA nanoparticles.

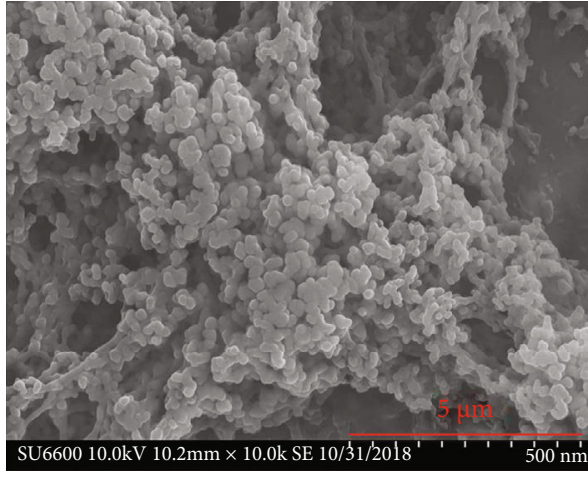

(a)

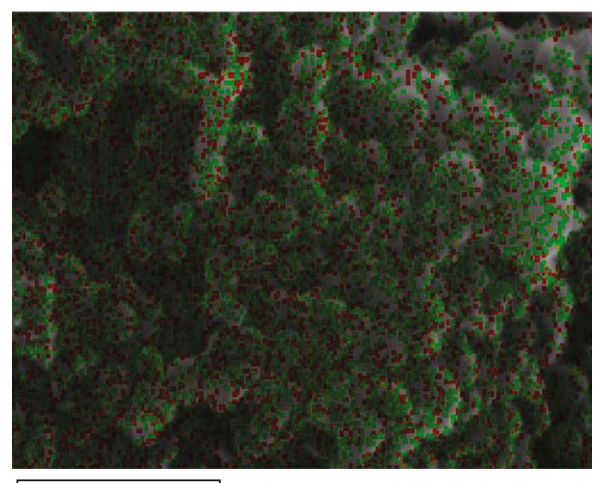

(c)

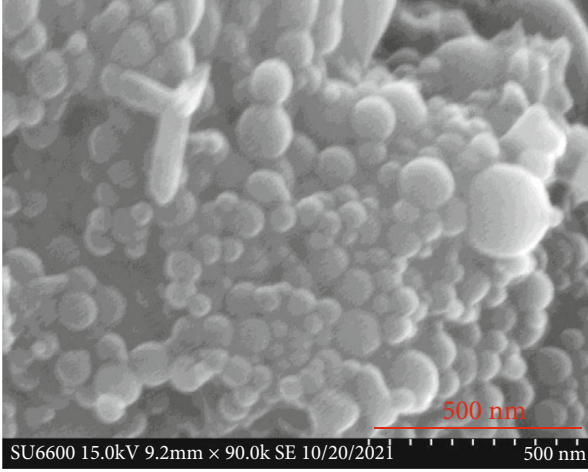

(b)

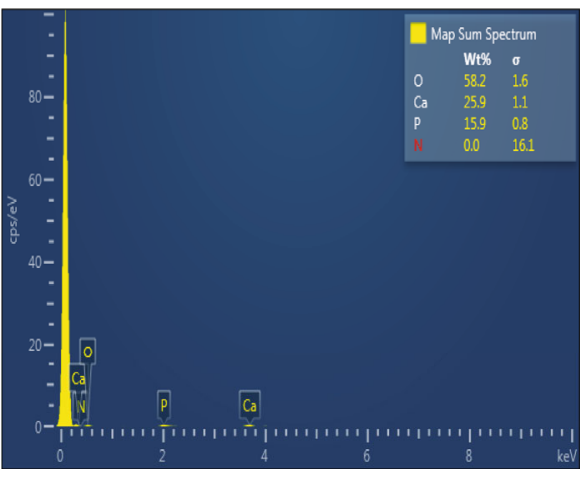

(d)

FIgURE 2: (a, b) SEM images of HP-55 coated HA nanoparticles. (c, d) Element mapping and respective EDX analysis.

spray, dip, and vacuum coating. It produces well covered uniform layer of coating material. Therefore, pharmacokineticrelated parameters such as dissolution and disintegration are less affected.

3.2. Thermal Analysis. Thermogravimetric analysis (Figure 3(c)) was carried out to assess any changes to the thermal stability of the raw materials when composited and to estimate the amount of VD loaded into the HA nanoparticle. The initial weight loss at temperatures below $100^{\circ} \mathrm{C}$ can be attributed to the elimination of surface-bound water. The mass loss due to the degradation of VD begins at $235^{\circ} \mathrm{C}$ in both the pure- and HA-bound samples [31]. The weight loss of the HA-VD indicates that up to $9.4 \%(w / w)$ of the final composite consists of VD alone. Moreover, the temperature at the maximum mass loss rate of HA-VD had shifted from 460 to $490^{\circ} \mathrm{C}$ compared to pure VD. The thermal degradation rate had also been reduced in the HA-VD composite in comparison. This signifies the increased thermal stability of the HA-VD composite, due to the presence of $\mathrm{HA}$ and its high inherent thermal stability.

3.3. Chemical Properties. The FTIR spectra of pure HA (A), pure VD (B), and HA-VD (C) are given in Figure 3(a). Theoretically, there are four $\mathrm{P}=\mathrm{O}$ vibrational modes present for the phosphate ions of HA, $v_{1}(980), v_{2}(470), v_{3}(1050,1085$, $1090)$, and $v_{4}(660,520)$. Out of these vibrational modes, $v_{3}$ is the most prominent peak; this appeared at $1030 \mathrm{~cm}^{-1}$ in 


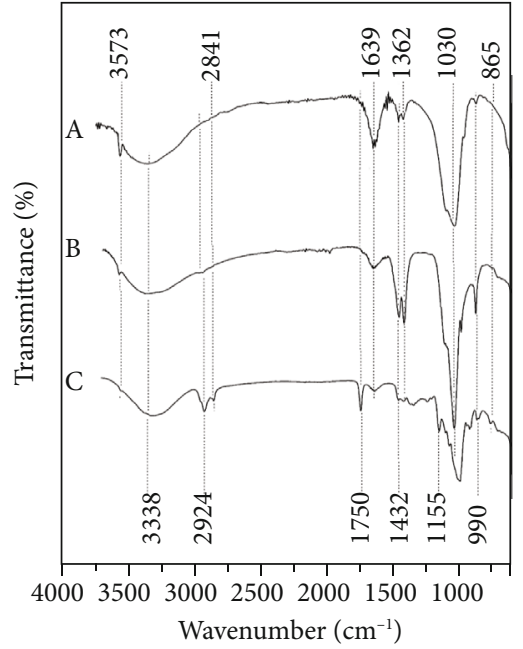

(a)

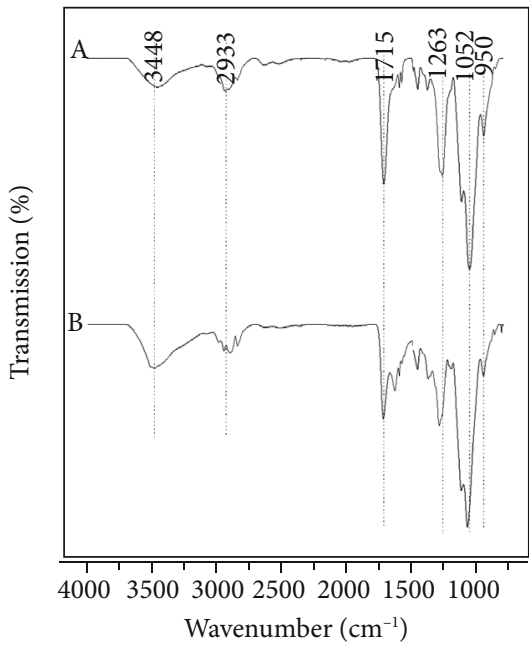

(b)

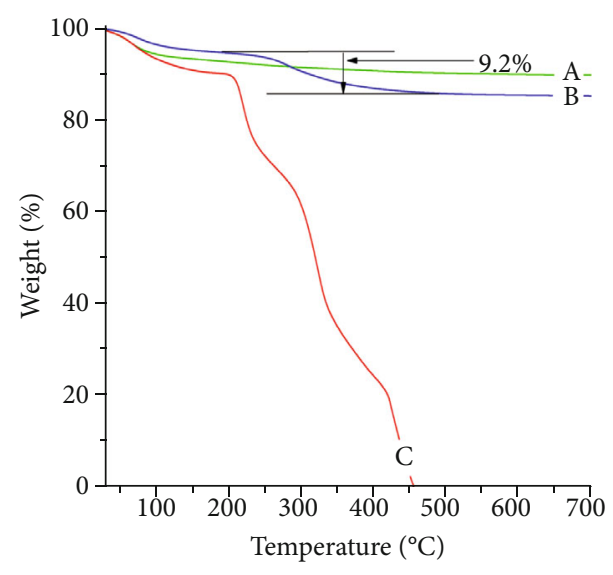

(c)

FIGURE 3: (a) FTIR spectrum of (A) HA, (B) VD, and (C) HA-VD. (b) FTIR spectrum of (A) pure HP-55 and (B) HP-55 coated HA-NP. (c) TGA of (A) HA, (B) HA-VD, and (C) VD.

the experimental spectra in combination with other two splitting. Additionally, there are two transmittance bands at 1362 and $1639 \mathrm{~cm}^{-1}$ due to the vibration of $\mathrm{CO}_{3}{ }^{2-}$. While pure HA cannot contain any $\mathrm{CO}_{3}{ }^{2-}$, traces may be observed due to difficulties in its removal following synthesis. The carbonate ion has a very strong transmittance, and the corresponding peaks are usually observed even at very low concentrations. The hydroxyl stretch is observed at $3338 \mathrm{~cm}^{-1}$, broadened due to the presence of intermolecular hydrogen bonding. There is an additional weak transmittance band at $3573 \mathrm{~cm}^{-1}$ characteristic of the vibration of $\mathrm{O}-\mathrm{H}$ (here present as $\left.\mathrm{Ca}(\mathrm{OH})_{2}\right)$. All of these peaks relevant to $\mathrm{HA}$ are present in the HA-VD composite [32,33].

The FTIR spectrum of pure VD (cholecalciferol) displays all major characteristic theoretical peaks. The strong band at $990 \mathrm{~cm}^{-1}$ is caused by the bending of $\mathrm{C}=\mathrm{C}$ alkene groups in the trans configuration, while the medium strength band at $1639 \mathrm{~cm}^{-1}$ is caused by the stretching of trans $\mathrm{C}=\mathrm{C}$ bonds. The peak at $1030 \mathrm{~cm}^{-1}$ is due to the stretching of the C-O bond of primary alcohols. It is a strong peak, but in the HA-VD spectrum, it overlaps with the $\mathrm{P}=\mathrm{O}$ vibrational peak of HA. VD also has a number of C-H alkane bonds, and the corresponding peaks for the bending vibration of these bonds appear at $1362 \mathrm{~cm}^{-1}$. The peak at $1432 \mathrm{~cm}^{-1}$ is due to the bending vibration of methyl C-H bonds, caused by four such methyl groups in the VD structure. The intensity of these two peaks (1362 and 1432) has reduced intensity in the composite spectrum due to the effect of HA overlaps. The characteristic broad peak at $3338 \mathrm{~cm}^{-1}$ is due to the presence of intermolecular $\mathrm{H}$-bonded $\mathrm{O}-\mathrm{H}$ stretching [33-35]. In the HA-VD spectrum, this peak also overlaps with the prominent hydroxyl peak of HA. Most of VD's characteristic IR peaks are observable in the HA-VD spectrum with minor shifts, indicating successful loading of VD onto HA. However, all peak intensities of the VD spectrum have been reduced due to the masking effect of HA, which makes up proportionally more of the composite than VD does.
The FTIR spectrum of HP-55 coated HA nanoparticle (Figure 3(b)) reveals that the HA nanoparticles are extensively covered by the polymeric material. All major peaks of pure HP-55 are also noticeable in HP-55 coated HA with slight deviation of the wavenumber. In pure HP-55, the major peaks are at 950,1052, 1263, 1715, 2933, and $3448 \mathrm{~cm}^{-1}$ while in HP-55 coated HA 950, 1072, 1286, 1720,2895 , and $3475 \mathrm{~cm}^{-1}$, respectively. This may be due to the presence of HA.

Both SEM images and FTIR data confirm the HP-55 coating of HA-VD nanoparticles. HP-55 is an acid stable polymer where only dissolve in basic $\mathrm{pH}$ range. HA undergoes acid degradation when the $\mathrm{pH}$ is lesser than 5 . Therefore, bare HA nanoparticles are not suitable for oral administration. However, successful coating of HP-55 indicates the protection of HA from gastric degradation, and HA nanoparticles release only within the small intestine where the $\mathrm{pH}$ is basic.

3.4. Cytocompatibility and Cell Penetration Studies. The cytotoxicity study was done using preosteoblast cells, which are precursors to osteoblasts. Osteoblasts are the major type of cells responsible for bone and cartilage regeneration. These cells also have a higher affinity for calcium and phosphorus and can absorb and accumulate these ions within the cytosol. The bare HA nanoparticle and its VD-loaded counterpart exhibit similar cytotoxicity profiles. Figure 4 shows the live cell assay images of nanomaterials at day 3 compared to the negative control. The lowest number of live cells was observed in HA-treated wells, but a significant difference was not observed between HA-VD and the negative control. According to the cell viability data (WST-1) after $24 \mathrm{~h}$ incubation, bare HA-treated cells showed $79 \pm 4 \%$ viability while HA-VD induced $85 \pm 5 \%$ viability relative to the negative control (100\%). The results indicate that both HA and HA-VD systems are nontoxic, as well as cytocompatible. However, bare HA appears slightly more toxic than VD- 


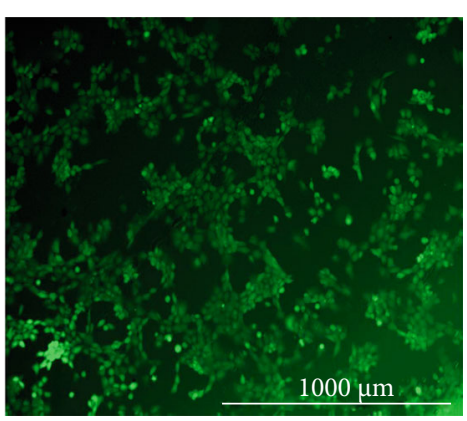

(a)

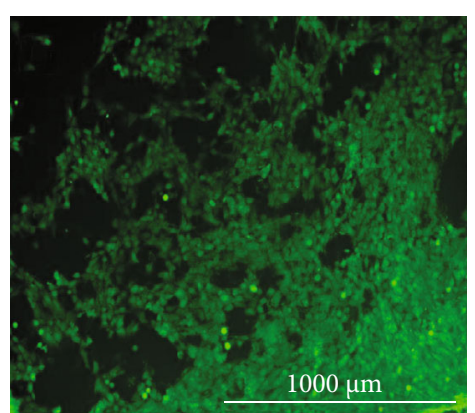

(b)

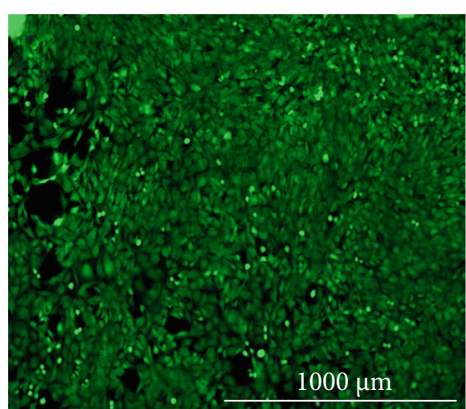

(c)

FIGURE 4: Live cell assay fluorescence images at day 3; green-live cells: (a) treated with HA; (b) treated with HA-VD; (c) negative control (cells only).

loaded HA. The cytotoxicity of HA mainly depends on size, shape, and the surface charge of the nanoparticle. Rod-, needle-, and oblong-shaped nanoparticles show higher toxicity profiles than spherical ones $[36,37]$. In this study, reduction of toxicity observed on the incorporation of VD may be due to the masking of HA's polar surface groups by $\mathrm{VD}$ or the replacement of some amount of HA by VD which is nontoxic.

The cell penetration study of HA was performed using the same cell line in order to identify the ability to enter HA-NPs through osteoblast cell membrane hence accumulation inside the cytosol. The concentration of $\mathrm{Ca}^{2+}$ in the supernatant of untreated samples was under the level of detection (LOD) of $1 \mu \mathrm{g} / \mathrm{mL}$, while treated samples showed a significantly high concentration $(22 \mu \mathrm{g} / \mathrm{mL})$ of $\mathrm{Ca}^{2+}$. The only possible cause of increasing intracellular $\mathrm{Ca}$ was the addition of HA-NP to the medium; the results suggest a high penetrative ability/uptake of HA-NP through the plasma membrane of osteoblast cells. In general, intracellular free $\mathrm{Ca}^{2+}$ concentration is significantly lower than extracellular concentration (according to published data, around 12,000x lower). Increase of intracellular free $\mathrm{Ca}^{2+}$ concentration may cause calcium dependent toxicities, hence reducing cell growth and differentiation, although the cytotoxicity study showed HA-NPs' apparent nontoxicity towards the tested preosteoblast cells. It can therefore be further concluded that the high level of intracellular $\mathrm{Ca}$ is mainly due to the HA-NPs acting as a $\mathrm{Ca}$ reservoir and maintaining a balance between free $\mathrm{Ca}^{2+}$ ion and insoluble Ca [38-40].

\subsection{UV-vis Characterization of VD and Drug Release Study.} Since VD is a highly lipophilic molecule, water solubility is minimal. Therefore, absolute ethanol was used as the solvent for UV-vis characterization. The UV-vis absorbance pattern of different concentrations of VD is shown in Figure 5(a). A uniform pattern of absorption maxima was identified between 200 and $300 \mathrm{~nm}$, and maximum absorbance $\left(\lambda_{\max }\right)$ was positioned within the $275-280 \mathrm{~nm}$ region. A bathochromic shift was observed proportional to the concentration. This may be due to the solvatochromism and interchanging bonds between solute and solvent. Also, there is a high probability of tiny micelle formation by the interaction of VD with solvent molecules [41, 42]. Therefore, concentration determinations were done by measuring absorbance between 275 and $280 \mathrm{~nm}$ rather than using a single $\lambda_{\max }$ value. The relevant standard curve for VD is shown in Figure 5(b). Linearity of absorbance of VD was observed within the $100 \mathrm{ppm}-1000 \mathrm{ppm}$ range. All concentration calculations for the release study were done based on this curve.

Figure 6 shows cumulative VD release percentage curves of the HA-VD nanocomposite at $37 \pm 0.5^{\circ} \mathrm{C}$ as a function of time. The trend of the graphs also fits with the typical diffusion of the small molecule. At day $1,35.4 \pm 3.2 \%(w / w)$ $(3.8 \mathrm{mg})$ of the loaded drug was released, and by day 10 , $86.7 \pm 5.6 \%(w / w)(9.2 \mathrm{mg})$ of the drug was released. There is a burst release of VD during the first two hours, and approximately, 25\% $(w / w)$ of VD is released within the first $30 \mathrm{~min}$. This is further evident from the drug release profile, where the first release phase (up to $2 \mathrm{~h}$ ) is ascribed to the rapid release of the surface-grafted VD and the second phase is attributed to the delayed release (up to 15 days) of the entrapped drug molecules within the HA nanoparticles. There is up to $15 \%(w / w)$ of unreleased VD even after the assessed period of release.

According to the release study, the amount of total encapsulated VD is $10.86 \mathrm{mg}(100 \%)$. Since the amount of HA-VD used for release study is $100 \mathrm{mg}$, the loading percentage of VD is $10.86 \%(w / w)$. The loading percentage given by the release study is close to the loading percentage given by TG analysis $(9.4 \% w / w)$. Low loading efficiency is commonly associated with VD delivery systems such as polymers, dendrimers, emulsions, and nanotubes. The highest loading efficiency observed in literature was encountered with liposomes and micelles, with efficiencies up to 40 and $55 \%$, respectively $[6,9]$.

The characteristic initial burst release can be another major pitfall since a large amount of VD is lost before reaching the target tissue [9]. Compared to other VD delivery methods, this HA-VD system shows a moderate first phase of the release profile equal to $25 \%$ of loaded VD released within the first five hours. Thereafter, the sustained release apparently maintains the serum VD level in a steady state. Conclusively, the release pattern represents typical VD requirements, and the transferable VD amount can be titrated by changing the loading percentages-by simply changing the VD:HA ratio.

There is a considerable body of extant literature on the topic of nanoparticle-based VD delivery systems. Among 


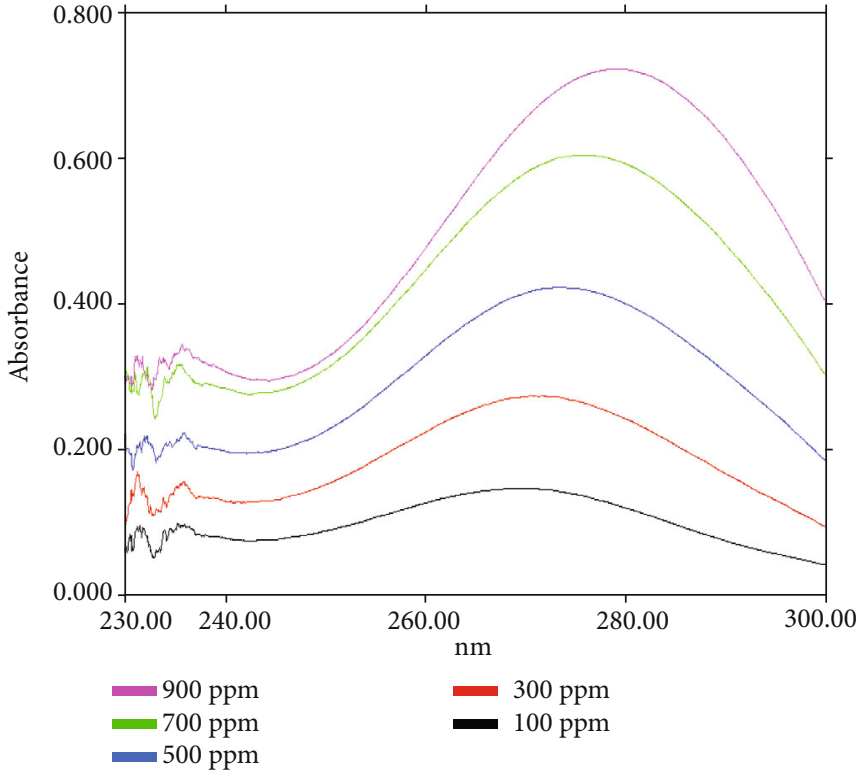

(a)

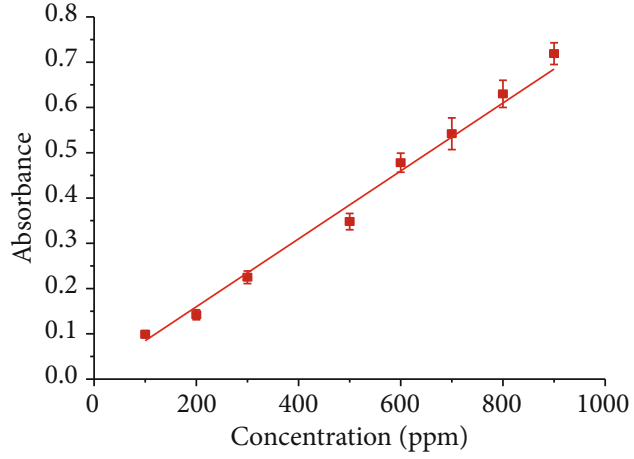

(b)

FIgURE 5: (a) UV-vis spectrum of VD at different concentrations in absolute ethanol. (b) Standard curve of VD based on UV-vis absorbance data.

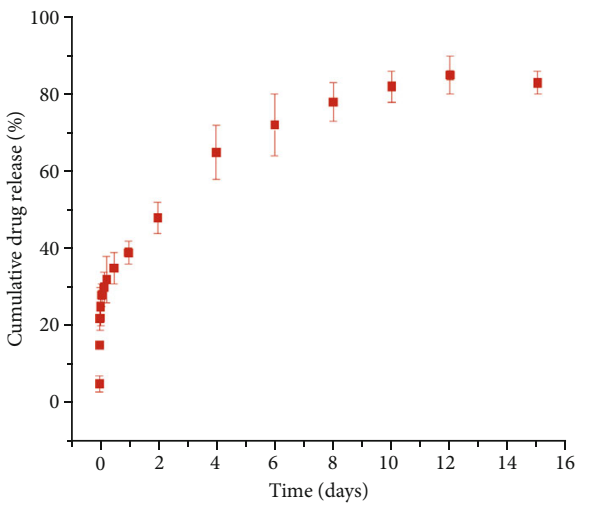

(a)

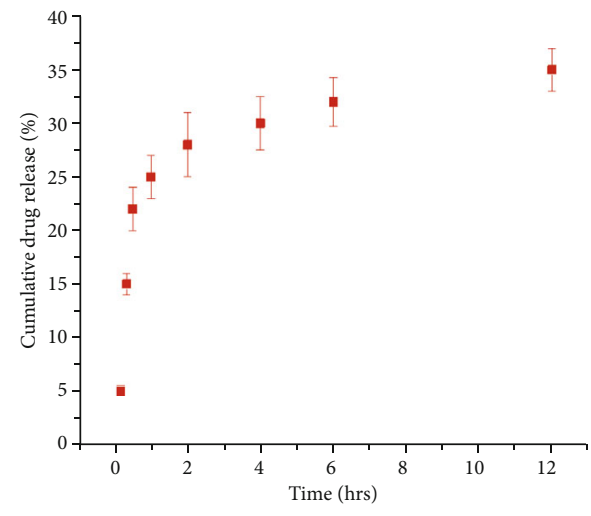

(b)

Figure 6: (a) Cumulative drug release percentage with respect to total amount of encapsulated drug; $n=3$. (b) Expanded version of cumulative drug release curve covering the first 12 hours.

them, biopolymeric nanoparticles [43-47], nanoemulsions [48-50], and colloidal systems [51] are prominent. Chitosan $[47,48]$, alginate [44], soy protein [46], polylactic acid [43], and PLGA [52-54] are the main types of materials incorporated with VD. VD delivery systems with inorganic nanoparticles appear not to be as popular. Ignjatović and colleagues prepared hydroxyapatite (HA) and PLGA-based nanoparticles for the local delivery of VD to enhance osteogenesis and bone tissue differentiation [52]. However, there is no literature examining oral delivery formulation of $\mathrm{VD}$ with solely HA-based nanoparticles. Hence, this is the first record of evaluating the applicability of HA nanoparticles on their own for oral delivery of $\mathrm{VD}$, also supplying $\mathrm{Ca}^{2+}$ and $\mathrm{PO}_{4}{ }^{3-}$ in one formulation to address a host of health problems in one package.

\section{Conclusions}

Nanoparticle-based drug delivery systems are a rapidly growing field, particularly making use of materials having biodegradable and biocompatible properties-hydroxyapatite is an example of such a candidate. Current vitamin D delivery systems are associated with various problems such as high burst release, accumulation inside adipose tissue, and suboptimal biocompatibility. In this study, HA was shown to be a good alternative for the oral delivery of $\mathrm{VD}$ due to its high affinity to bone tissue. Further, HA also acts as a source of $\mathrm{Ca}_{3}\left(\mathrm{PO}_{4}\right)_{2}$, and the HA-VD composite synthesized herein is thus a type of codelivery system, providing both VD and $\mathrm{Ca}_{3}\left(\mathrm{PO}_{4}\right)_{2}$. HA-VD nanomaterials showed an extended release profile for ten days, and the particles 
were found to be biocompatible. Electrospray was used to coat HA with the enteric polymer HP-55, generating wellcovered particles resistant to gastric $\mathrm{pH}$. This study suggests the use of HP-55 coated VD-loaded HA nanoparticles as a potent alternative for sustained and targeted oral delivery of $\mathrm{VD}$ with $\mathrm{Ca}^{2+}$ and $\mathrm{PO}_{4}{ }^{3-}$.

\section{Data Availability}

The data generated through the study given in main text and as a supplementary material. Further clarification and information are available upon request from the corresponding author.

\section{Conflicts of Interest}

The authors declare that they have no known competing financial interests or personal relationships that could have appeared to influence the work reported in this paper.

\section{Funding}

The research was financially assisted by the university research grant ASP/01/RE/AHS/2021/90.

\section{Supplementary Materials}

Supplementary Materials SEM and TEM images of synthesized nanoparticles and ICP-MS data are given as supplementary material. (Supplementary Materials)

\section{References}

[1] H. F. DeLuca, "History of the discovery of vitamin D and its active metabolites," BoneKEy reports, vol. 3, p. 479, 2014.

[2] B. Metabolism, "Anti-diabetic and toxic effects of vanadium compounds," Endocrinology, vol. 206, pp. 1159-1164, 1996.

[3] P. R. Ebeling, "Vitamin D and bone health: epidemiologic studies," BoneKEy reports, vol. 3, p. 511, 2014.

[4] E. Laird, M. Ward, E. McSorley, J. J. Strain, and J. Wallace, "Vitamin D and bone health; potential mechanisms," Nutrients, vol. 2, no. 7, pp. 693-724, 2010.

[5] J. C. Gallagher, V. Yalamanchili, and L. M. Smith, "The effect of vitamin $\mathrm{D}$ on calcium absorption in older women," The Journal of Clinical Endocrinology and Metabolism, vol. 97, no. 10, pp. 3550-3556, 2012.

[6] E. Glowka, J. Stasiak, and J. Lulek, "Drug delivery systems for vitamin D supplementation and therapy," Pharmaceutics, vol. 11, no. 7, p. 347, 2019.

[7] I. Torjesen, "Vitamin D supplements do not protect bone health, analysis finds," BMJ, vol. 363, article k4223, 2018.

[8] T. Dinizulu, D. Griffin, J. Carey, and E. Mulkerrin, "Vitamin D supplementation versus combined calcium and vitamin D in older female patients - an observational study," The journal of nutrition, health \& aging, vol. 15, no. 8, pp. 605-608, 2011.

[9] M. J. Ramalho, M. A. N. Coelho, and M. C. Pereira, "Nanoparticles for delivery of vitamin D: challenges and opportunities," A Critical Evaluation of Vitamin D - Clinical Overview, vol. 11, pp. 231-249, 2017.
[10] M. F. Holick, "Vitamin D - effects on skeletal and extraskeletal health and the need for supplementation," Nutrients, vol. 2013, pp. 155-168, 2016.

[11] S. J. Chon, Y. K. Koh, J. Y. Heo et al., "Effects of vitamin D deficiency and daily calcium intake on bone mineral density and osteoporosis in Korean postmenopausal woman," Obstetrics \& Gynecology Science, vol. 60, no. 1, pp. 53-62, 2017.

[12] M. C. Silva and T. W. Furlanetto, "Intestinal absorption of vitamin D: a systematic review," Nutrition Reviews, vol. 76, no. 1, pp. 60-76, 2018.

[13] V. K. Maurya and M. Aggarwal, "Factors influencing the absorption of vitamin D in GIT: an overview," Journal of Food Science and Technology, vol. 54, no. 12, pp. 3753-3765, 2017.

[14] S. Christakos, P. Dhawan, A. Porta, L. J. Mady, and T. Seth, "Vitamin D and intestinal calcium absorption," Molecular and Cellular Endocrinology, vol. 347, no. 1-2, pp. 25-29, 2011.

[15] T. J. Levingstone, S. Herbaj, and N. J. Dunne, "Calcium phosphate nanoparticles for therapeutic applications in bone regeneration," Nanomaterials, vol. 9, no. 11, p. 1570, 2019.

[16] N. Khazai, S. E. Judd, and V. Tangpricha, "Calcium and vitamin D: skeletal and extraskeletal health," Current Rheumatology Reports, vol. 10, no. 2, pp. 110-117, 2008.

[17] J. A. Sunyecz, "The use of calcium and vitamin D in the management of osteoporosis," Therapeutics and Clinical Risk Management, vol. 4, no. 4, pp. 827-836, 2008.

[18] U. Trautvetter, N. Neef, M. Leiterer, M. Kiehntopf, J. Kratzsch, and G. Jahreis, "Effect of calcium phosphate and vitamin $\mathrm{D}_{3}$ supplementation on bone remodelling and metabolism of calcium, phosphorus, magnesium and iron," Nutrition Journal, vol. 13, no. 1, pp. 1-9, 2014.

[19] D. R. K. Weerasuriya, W. P. S. L. Wijesinghe, and R. M. G. Rajapakse, "Encapsulation of anticancer drug copper bis(8hydroxyquinoline) in hydroxyapatite for $\mathrm{pH}$-sensitive targeted delivery and slow release," Materials Science and Engineering: C, vol. 71, pp. 206-213, 2017.

[20] W. P. S. L. Wijesinghe, M. M. M. G. P. G. Mantilaka, E. V. A. Premalal et al., "Facile synthesis of both needle-like and spherical hydroxyapatite nanoparticles: effect of synthetic temperature and calcination on morphology, crystallite size and crystallinity," Materials Science and Engineering: C, vol. 42, pp. 83-90, 2014.

[21] R. T. De Silva, R. K. Dissanayake, M. P. Mantilaka et al., "Drug-loaded halloysite nanotube-reinforced electrospun alginate-based nanofibrous scaffolds with sustained antimicrobial protection," ACS applied materials \& interfaces, vol. 10, no. 40, pp. 33913-33922, 2018.

[22] Y. Lu, J. Huang, G. Yu et al., "Coaxial electrospun fibers: applications in drug delivery and tissue engineering," NanoBiotechnology, vol. 8, no. 5, pp. 654-677, 2016.

[23] B. Pant, M. Park, and S. J. Park, "Drug delivery applications of core-sheath nanofibers prepared by coaxial electrospinning: a review," Pharmaceutics, vol. 11, no. 7, p. 305, 2019.

[24] E. Hasanvand, M. Fathi, and A. Bassiri, "Production and characterization of vitamin $\mathrm{D}_{3}$ loaded starch nanoparticles: effect of amylose to amylopectin ratio and sonication parameters," Journal of Food Science and Technology, vol. 55, no. 4, pp. 1314-1324, 2018.

[25] T. N. Orlova and I. P. Terenetskaya, "Possible use of provitamin $\mathrm{D}_{3}$ photoisomerization for spectral dosimetry of bioactive antirachitic UV radiation," Journal of Applied Spectroscopy, vol. 76, no. 2, pp. 240-244, 2009. 
[26] J. M. Unagolla and A. C. Jayasuriya, "Enhanced cell functions on graphene oxide incorporated 3D printed polycaprolactone scaffolds," Materials Science and Engineering: C, vol. 102, pp. 1-11, 2019.

[27] W. P. T. D. Perera, R. K. Dissanayake, U. I. Ranatunga et al., "Curcumin loaded zinc oxide nanoparticles for activityenhanced antibacterial and anticancer applications," RSC Advances, vol. 10, no. 51, pp. 30785-30795, 2020.

[28] M. P. Nikolova and M. S. Chavali, "Metal oxide nanoparticles as biomedical materials," Biomimetics, vol. 5, no. 2, p. 27, 2020.

[29] Y. Cai, Y. Liu, W. Yan et al., "Role of hydroxyapatite nanoparticle size in bone cell proliferation," Journal of Materials Chemistry, vol. 17, no. 36, pp. 3780-3787, 2007.

[30] D. S. Gunasekera, B. P. Senanayake, R. K. Dissanayake et al., "Rapid Detection Method to Quantify Linamarin Content in Cassava," Journal of Bioprocessing and Biotechniques, vol. 8, pp. 1-7, 2018.

[31] M. Zareie, A. Abbasi, and S. Faghih, "Thermal stability and kinetic study on thermal degradation of vitamin $\mathrm{D}_{3}$ in fortified canola oil," Journal of Food Science, vol. 84, no. 9, pp. 24752481, 2019.

[32] I. Rehman and W. Bonfield, "Characterization of hydroxyapatite and carbonated apatite by photo acoustic FTIR spectroscopy," Journal of Materials Science. Materials in Medicine, vol. 8, no. 1, pp. 1-4, 1997.

[33] A. Ślósarczyk, Z. Paszkiewicz, and C. Paluszkiewicz, "FTIR and XRD evaluation of carbonated hydroxyapatite powders synthesized by wet methods," Journal of Molecular Structure, vol. 744-747, pp. 657-661, 2005.

[34] A. Heidari, "A literature review of malaria intervention in Zanzibar," Journal of Biometrics and Biostatistics, vol. 7, no. 4, pp. 3-5, 2016.

[35] M. Tabibian, M. Torbati, M. R. A. Mogaddam, M. Mirlohi, M. Sadeghi, and J. Mohtadinia, "Evaluation of vitamin $\mathrm{D}_{3}$ and $\mathrm{D}_{2}$ stability in fortified flat bread samples during dough fermentation, baking and storage," Advanced Pharmaceutical Bulletin, vol. 7, no. 2, pp. 323-328, 2017.

[36] X. Zhao, S. Ng, B. C. Heng et al., "Cytotoxicity of hydroxyapatite nanoparticles is shape and cell dependent," Archives of Toxicology, vol. 87, no. 6, pp. 1037-1052, 2013.

[37] X. Liu and J. Sun, "Potential proinflammatory effects of hydroxyapatite nanoparticles on endothelial cells in a monocyte-endothelial cell coculture model," International Journal of Nanomedicine, vol. 9, pp. 1261-1273, 2014.

[38] V. Veldurthy, R. Wei, L. Oz, P. Dhawan, Y. H. Jeon, and S. Christakos, "Vitamin D, calcium homeostasis and aging," Bone Res., vol. 4, no. 1, pp. 1-7, 2016.

[39] M. Peacock, "Calcium metabolism in health and disease," Clinical Journal of the American Society of Nephrology, vol. 5, Supplement 1, pp. S23-S30, 2010.

[40] F. Pu, N. Chen, and S. Xue, "Calcium intake, calcium homeostasis and health," Food Sci. Hum. Wellness, vol. 5, no. 1, pp. 8$16,2016$.

[41] A. Albeck, N. Friedman, M. Sheves, and M. Ottolenghi, "Factors affecting the absorption maxima of acidic forms of bacteriorhodopsin. A study with artificial pigments," Biophysical Journal, vol. 56, no. 6, pp. 1259-1265, 1989.

[42] S. Kumar, "BUCHBESPRECHUNG," Limnologica, vol. 36, no. 1, p. $66,2006$.
[43] E. Almouazen, S. Bourgeois, L. P. Jordheim, H. Fessi, and S. Briançon, "Nano-encapsulation of vitamin $\mathrm{D}_{3}$ active metabolites for application in chemotherapy: formulation study and in vitro evaluation," Pharmaceutical Research, vol. 30, no. 4, pp. 1137-1146, 2013.

[44] C. Banville, J. C. Vuillemard, and C. Lacroix, "Comparison of different methods for fortifying Cheddar cheese with vitamin D," International Dairy Journal, vol. 10, no. 5-6, pp. 375$382,2000$.

[45] Q. Li, C. G. Liu, Z. H. Huang, and F. F. Xue, "Preparation and characterization of nanoparticles based on hydrophobic alginate derivative as carriers for sustained release of vitamin $\mathrm{D}_{3}$," Journal of Agricultural and Food Chemistry, vol. 59, no. 5, pp. 1962-1967, 2011.

[46] Z. Teng, Y. Luo, and Q. Wang, "Carboxymethyl chitosan-soy protein complex nanoparticles for the encapsulation and controlled release of vitamin $\mathrm{D}_{3}$," Food Chemistry, vol. 141, no. 1, pp. 524-532, 2013.

[47] A. Abbasi, Z. Emam-Djomeh, M. A. E. Mousavi, and D. Davoodi, "Stability of vitamin $\mathrm{D}_{3}$ encapsulated in nanoparticles of whey protein isolate," Food Chemistry, vol. 143, pp. 379-383, 2014.

[48] W. Li, H. Peng, F. Ning et al., "Amphiphilic chitosan derivative-based core-shell micelles: synthesis, characterisation and properties for sustained release of vitamin $\mathrm{D}_{3}$," Food Chemistry, vol. 152, pp. 307-315, 2014.

[49] M. Tippetts, S. Martini, C. Brothersen, and D. J. McMahon, "Fortification of cheese with vitamin $\mathrm{D}_{3}$ using dairy protein emulsions as delivery systems," Journal of Dairy Science, vol. 95, no. 9, pp. 4768-4774, 2012.

[50] Y. Luo, Z. Teng, and Q. Wang, "Development of zein nanoparticles coated with carboxymethyl chitosan for encapsulation and controlled release of vitamin D3," Journal of Agricultural and Food Chemistry, vol. 60, no. 3, pp. 836-843, 2012.

[51] K. Ziani, Y. Fang, and D. J. McClements, "Encapsulation of functional lipophilic components in surfactant-based colloidal delivery systems: vitamin E, vitamin D, and lemon oil," Food Chemistry, vol. 134, no. 2, pp. 1106-1112, 2012.

[52] N. Ignjatović, V. Uskoković, Z. Ajduković, and D. Uskoković, "Multifunctional hydroxyapatite and poly(d,l-lactide-co-glycolide) nanoparticles for the local delivery of cholecalciferol," Materials Science and Engineering: C, vol. 33, no. 2, pp. 943950, 2013.

[53] J. C. Bonor, R. J. Schaefer, N. Menegazzo, K. Booksh, and A. G. Nohe, "Design of 1,25 dihydroxyvitamin D3 coupled quantum dots, a novel imaging tool," Journal of Nanoscience and Nanotechnology, vol. 12, no. 3, pp. 2185-2191, 2012.

[54] H. Maeda, K. Tsukigawa, and J. Fang, "A retrospective 30 years after discovery of the enhanced permeability and retention effect of solid tumors: next-generation chemotherapeutics and photodynamic therapy-problems, solutions, and prospects," Microcirculation, vol. 23, no. 3, pp. 173-182, 2016. 